\title{
GORENSTEIN PROPERTY OF HOPF GRADED ALGEBRAS
}

\author{
Q.-S. WU \\ Institute of Mathematics, Fudan University, Shanghai, 200433, China \\ e-mail:qswu@fudan.edu.cn \\ and J. J. ZHANG \\ Department of Mathematics, Box 354350, University of Washington, Seattle, WA 98195, USA \\ e-mail: zhang@math.washington.edu
}

(Received 30 July, 2001; accepted 15 February, 2002)

\begin{abstract}
Let $A$ be a locally finite, $\mathbb{N}$-graded, noetherian algebra with a balanced dualizing complex. If $A$ is a Hopf algebra, then $A$ has finite injective dimension.
\end{abstract}

2000 Mathematics Subject Classification. 16E10, 16W30, 16W50.

In [2] and [3] Brown and Goodearl showed that a noetherian affine polynomial identity (PI) Hopf algebra with finite injective dimension has various good homological properties. They verified that many examples of noetherian affine PI Hopf algebras, some of which are quantum groups at roots of unity, have finite injective dimension. In [2, Question A] Brown asks if every noetherian affine PI Hopf algebra has finite left and right injective dimension. Recently we gave an affirmative answer to Brown's question in $[9,0.1]$.

In this short note we are trying to provide some evidence that a noetherian affine Hopf algebra, not necessarily PI, has finite injective dimension. We prove the following statement.

THEOREM 1. Let $A$ be a locally finite, $\mathbb{N}$-graded, noetherian algebra with a balanced dualizing complex. If $A$ is a Hopf algebra, then $A$ has finite left and right injective dimension and satisfies the AS-Gorenstein condition.

The AS-Gorenstein condition is defined after Definition 2. The existence of dualizing complexes over various graded rings was studied in [10] and some of which are listed in Corollary 5 below. The proof of Theorem 1 is given at the end. We refer to [5] for the definition of Hopf algebra. Note that we don't assume that $A$ is connected graded nor that the coalgebra structure is compatible with the grading. The statement is still limited because of the graded hypothesis. It would be nice if the graded hypothesis could be deleted.

Let $k$ be a base field. Let $A$ be locally finite and $\mathbb{N}$-graded over $k$, namely, $A=\bigoplus_{i=0}^{\infty} A_{i}$ and $\operatorname{dim}_{k} A_{i}<\infty$ for all $i$. Sometimes we will simply say $A$ is graded. Usually an $A$-module means a $\mathbb{Z}$-graded left $A$-module. Let $A^{\circ}$ denote the opposite ring of $A$. An $A^{\circ}$-module means a $\mathbb{Z}$-graded right $A$-module. An $A \otimes A^{\circ}$-module means a $\mathbb{Z}$-graded $A$-bimodule. An $A$-module is said to be finite if it is finitely generated.

First we recall some definitions and results due to Yekutieli [10] and Van den Bergh [6]. Other definitions, results and notations can be found in [6], [7] and [10]. Let $\mathrm{D}_{f}^{b}(A)$ 
be the derived category of bounded complexes of left graded $A$-modules with finite cohomological modules.

Definition 2 [10,3.3]. A complex $R \in \mathrm{D}^{b}\left(A \otimes A^{\circ}\right)$ is called dualizing if it satisfies the following conditions:

(2.1) $R$ has finite graded injective dimension over $A$ and over $A^{\circ}$;

(2.2) $R$ has finite cohomologies over $A$ and over $A^{\circ}$;

(2.3) The canonical morphisms $A \rightarrow \operatorname{RHom}_{A}(R, R)$ and $A \rightarrow \operatorname{RHom}_{A^{\circ}}(R, R)$ are isomorphisms in $\mathrm{D}\left(A \otimes A^{\circ}\right)$.

If $A$ is noetherian and has finite left and right graded injective dimension, then (2.1) holds for $R:=A$. The conditions (2.2) and (2.3) are automatic in this case. Therefore $R=A$ is a dualizing complex. We say $A$ satisfies the AS-Gorenstein condition (where AS stands for Artin and Schelter) if $A$ has finite left and right graded injective dimension, say $d$, and for any graded simple $A$-module $M, \operatorname{Ext}_{A}^{i}(M, A)=0$ for $i \neq d$ and $\operatorname{Ext}_{A}^{d}(M, A)$ is a graded simple $A^{\circ}$-module.

Graded rings with balanced dualizing complexs (see Definition 3) can be viewed as a generalization of AS-Gorenstein rings. To define the balanced condition we need recall several basic notions. Let $A$ be a left noetherian graded algebra and let $\mathfrak{m}$ be the graded Jacobson radical of $A$. We say $A$ satisfies the left $\chi$-condition if $\operatorname{Ext}_{A}^{i}(A / \mathfrak{m}, M)$ is finite dimensional over $k$ for all $i$ and all graded finite $A$-modules $M$. The right $\chi$-condition is defined similarly. If $A$ is noetherian and satisfies both left and right $\chi$-condition, we say $A$ satisfies the $\chi$-condition.

For any graded $A$-module $M$, the $\mathfrak{m}$-torsion functor $\Gamma_{\mathfrak{m}}$ is defined to be

$$
\Gamma_{\mathfrak{m}}(M)=\left\{x \in M \mid \mathfrak{m}^{n} x=0, \forall n \gg 0\right\} .
$$

The systems $\left\{\mathfrak{m}^{n}\right\}$ and $\left\{A_{\geq n}\right\}$ are cofinal to each other and $\Gamma_{\mathfrak{m}}(M)$ is the union of the finite dimensional submodules of $M$. The derived functor $\mathrm{R} \Gamma_{\mathfrak{m}}$ is defined on the derived category $\mathrm{D}^{+}(A)$. For any complex $X \in \mathrm{D}^{+}(A)$, the $i$ th local cohomology is defined to be

$$
\mathrm{H}_{\mathfrak{m}}^{i}(X)=\mathrm{R}^{i} \Gamma_{\mathfrak{m}}(X)
$$

The left cohomological dimension of $A$ (or of $\Gamma_{\mathfrak{m}}$ ) is

$$
c d(A)=\sup \left\{i \mid \mathrm{H}_{\mathfrak{m}}^{i}(M) \neq 0, \text { for all graded } A \text {-modules } M\right\} .
$$

If $M$ is a graded module, let $M^{\prime}$ denote the graded dual of $M$, namely,

$$
M^{\prime}=\bigoplus_{n \in \mathbb{Z}} \operatorname{Hom}_{k}\left(M_{-n}, k\right)
$$

The graded dual $(-)^{\prime}$ can be applied to complexes too. Let $A^{\prime}$ be the graded dual of the bimodule $A$. Then $A^{\prime}$ is an $A$-bimodule and ${ }_{A} A^{\prime}$ is the injective hull of the graded $A$-module $A / \mathfrak{m}$.

Definition $3[\mathbf{1 0}, 4.1]$. A dualizing complex $R$ over $A$ is called balanced if there are isomorphisms

$$
\mathrm{R} \Gamma_{\mathfrak{m}}(R) \cong \mathrm{R} \Gamma_{\mathfrak{m}^{\circ}}(R) \cong A^{\prime}
$$

in $\mathrm{D}\left(A \otimes A^{\circ}\right)$. 
If a balanced dualizing complex over $A$ exists, then it is unique and has nice functorial properties. The next result of Van den Bergh says that there is a natural way to construct the balanced dualizing complex. Hence the balanced dualizing complex over $A$ is viewed as a natural object associated to $A$.

THEOREM 4 [6, 6.3 and 5.1]. Let $A$ be a noetherian graded algebra.

(1) A admits a balanced dualizing complex if and only if $A$ satisfies the following two conditions:

(4.1) A satisfies the $\chi$-condition, and

(4.2) A has finite left and right cohomological dimension.

(2) If $A$ admits a balanced dualizing complex $R$, then

$$
R=\left(\mathrm{R} \Gamma_{\mathfrak{m}} A\right)^{\prime} .
$$

(3) If $M$ is a graded A-module, then

$$
\mathrm{R} \Gamma_{\mathfrak{m}}(M)^{\prime} \cong \operatorname{RHom}_{A}(M, R) .
$$

The original results of Van den Bergh were stated for connected graded rings, but his proofs work for locally finite $\mathbb{N}$-graded rings. The same results were proved in the semilocal case in a slightly more general setting (see [4] and [8]).

The existence of balanced dualizing complex is considered to be a mild condition and it can be verified for PI rings, (graded) FBN rings and rings having enough normal elements. The definition of a graded FBN ring can be found in [1, p. 286]. Recall that a graded ring $A$ has enough normal elements if every non-artinian graded prime factor $A / \mathfrak{p}$ contains a normal element of positive degree [11, p. 36]. Therefore we have the following corollary to Theorems 1 and 4 .

Corollary 5. Let $A$ be a locally finite, $\mathbb{N}$-graded, noetherian algebra. Suppose either $A$ is graded $\mathrm{FBN}$ (e.g. FBN, PI), or A has enough normal elements. If $A$ is a Hopf algebra, then $A$ has finite injective dimension and satisfies the AS-Gorenstein condition.

Proof. By [1, 8.8 and 8.13] $A$ satisfies (4.1) and (4.2). Theorem 4 implies that $A$ has a balanced dualizing complex. Now the assertion follows from Theorem 1 .

We need a few lemmas for the proof of Theorem 1. Let injdim denote the graded injective dimension.

LEMMA 6. Let $A$ be a graded noetherian algebra with a balanced dualizing complex $R$.

(1) If $M$ is a graded $A$-module and $\operatorname{dim}_{k} M<\infty$, then $\operatorname{Ext}_{A}^{i}(M, R)=0$ for all $i \neq 0$ and $\operatorname{Ext}_{A}^{0}(M, R) \cong M^{\prime}$.

(2) $\operatorname{injdim}_{A} R=\operatorname{injdim} R_{A}=0$.

Proof. (1). By the definition of the balanced condition (3.1) $\operatorname{Ext}_{A}^{i}(M, R)=0$ for all $i \neq 0$ and

$$
\operatorname{Ext}_{A}^{0}(M, R) \cong \operatorname{Hom}_{A}\left(M, A^{\prime}\right) \cong \operatorname{Hom}_{k}(M, k)=M^{\prime} .
$$

(2). By (1) it remains to show that $\operatorname{Ext}_{A}^{i}(M, R)=0$ for all graded $A$-modules $M$ and for all $i>0$. By (4.4),

$$
\operatorname{Ext}_{A}^{i}(M, R) \cong\left(R^{-i} \Gamma_{\mathfrak{m}}(M)\right)^{\prime}=(0)^{\prime}=0
$$

for all $i>0$. 
Let $A$ be a Hopf algebra over $k$, which is not necessarily graded. Let $k$ also denote the trivial module $A / \epsilon^{-1}(0)$ where $\epsilon: A \rightarrow k$ is the counit of $A$. The following lemma is proved by Brown and Goodearl [3, 1.11]. A proof is also given in [7, 4.8].

Lemma 7. Let $A$ be a Hopf algebra and let $d:=\inf \left\{i \mid \operatorname{Ext}_{A}^{i}(k, A) \neq 0\right\}$.

(1) For every nonzero finite dimensional $A$-module $V, d=\inf \left\{i \mid \operatorname{Ext}_{A}^{i}(V, A) \neq 0\right\}$.

(2) $\operatorname{Ext}_{A}^{d}(-, A)$ is exact on finite dimensional modules.

We now go back to graded rings. The next lemma is a graded version of [7, 4.2(3)] and its proof is the same as the proof of [7, 4.2(3)].

LEMmA 8. Let $\Omega$ be a finite graded $A$-module. If $\operatorname{Hom}_{A}(\Omega,-)$ is an exact functor on finite dimensional graded A-modules, then $\Omega$ is graded projective.

Proof of Theorem 1. Let $A$ be a graded algebra with a balanced dualizing complex $R$. Suppose $A$ is a Hopf algebra. Let $d$ be as in Lemma 7. By Lemma 7(1),

$$
d=\min \left\{i \mid \operatorname{Ext}_{A}^{i}(A / \mathfrak{m}, A) \neq 0\right\} .
$$

Hence $\mathrm{R}^{i} \Gamma_{\mathfrak{m}}(A)=0$ for all $i<d$ and $\mathrm{R}^{d} \Gamma_{\mathfrak{m}}(A) \neq 0$. It follows from (4.3) that $h^{i}(R)=0$ for all $i>-d$ and $\Omega:=h^{-d}(R) \neq 0$. Therefore, for any graded $A$-module $M$,

$$
\operatorname{Ext}_{A}^{d}(R, M) \cong \operatorname{Hom}_{A}(\Omega, M) .
$$

Let $F$ be the duality functor $\mathrm{RHom}_{A}(-, R)$. If $N$ is a finite dimensional graded right $A$-module, then $F(N) \cong N^{\prime}$ (see Lemma 6(1)) and

$$
\operatorname{Ext}_{A^{\circ}}^{d}\left(N, A_{A}\right) \cong \operatorname{Ext}_{A}^{d}(F(A), F(N)) \cong \operatorname{Ext}_{A}^{d}\left(R, N^{\prime}\right) \cong \operatorname{Hom}_{A}\left(\Omega, N^{\prime}\right) .
$$

It follows from Lemma 7(1) that the left-most term of (9.1) is exact on finite dimensional modules $N$. By (9.1) and the fact that $(-)^{\prime}$ is exact, the right-most term of (9.1) is exact on finite dimensional modules $M:=N^{\prime}$, namely, the functor $\operatorname{Hom}_{A}(\Omega,-)$ is exact on finite-dimensional graded left $A$-modules. By the definition of dualizing complex (2.2), $\Omega$ is finite over $A$ and over $A^{\circ}$. By Lemma $8, \Omega$ is graded projective. By Lemma 7(1) and (9.1), $\operatorname{Hom}(\Omega, M) \neq 0$ for every nonzero finite-dimensional $A$ module $M$. Hence $\Omega$ is a graded progenerator. Since $R$ is bounded above at the position $-d, R \cong X \oplus \Omega[d]$ where $X$ is bounded above at $-d-1$. If $X$ is not quasiisomorphic to 0 , then $\operatorname{Ext}_{A}^{-i}(\Omega[d], X) \neq 0$ for some $i>0$, which gives a nonzero element in $\operatorname{Ext}_{A}^{-i}(R, R)$ for some $i>0$. This contradicts the fact $\operatorname{Ext}_{A}^{-i}(R, R)=0$ for all $i \neq 0$ (see (2.3)). Therefore we obtain that $R=\Omega[d]$. By Lemma 6(2), injdim ${ }_{A} R=0$, which implies that $\operatorname{injdim}_{A} \Omega=d$.

The right-hand versions of these statements hold too.

Since $\Omega$ is a progenerator, the injective dimension of ${ }_{A} A$ is equal to the injective dimension of ${ }_{A} \Omega$, which is $d$. Similarly injdim ${ }_{A} A=d$.

By (4.3),

$$
R \Gamma_{\mathfrak{m}}(A)=(R)^{\prime} \cong(\Omega[d])^{\prime}=\Omega^{\prime}[-d] .
$$

Since $\Omega_{A}$ is a progenerator, ${ }_{A} \Omega^{\prime}$ is a cofinitely generated injective cogenerator. For any finite dimensional module $M$,

$$
\mathrm{RHom}_{A}(M, A)=\mathrm{RHom}_{A}\left(M, \mathrm{R} \Gamma_{\mathfrak{m}}(A)\right) \cong \operatorname{RHom}_{A}\left(M, \Omega^{\prime}[-d]\right) .
$$


Hence $\operatorname{Ext}^{i}(M, A)=0$ for all $i \neq d$ and $\operatorname{Ext}_{A}^{d}(M, A) \cong \operatorname{Hom}_{A}\left(M, \Omega^{\prime}\right)$. In particular, $\operatorname{Ext}_{A}^{d}(M, A)$ is finite dimensional and $\operatorname{Ext}_{A}^{d}(-, A)$ is exact on finite dimensional $A$-modules. Since $A$ is a dualizing complex (not balanced though), the double-Ext spectral sequence $[11,1.7]$

$$
\operatorname{Ext}_{A^{\circ}}^{p}\left(\operatorname{Ext}_{A}^{-q}(M, A), A\right) \Rightarrow M
$$

shows that the functors $\operatorname{Ext}_{A}^{d}(-, A)$ and $\operatorname{Ext}_{A^{\circ}}^{d}(-, A)$ induce a duality between the category of finite dimensional graded left $A$-modules and that of right modules. Therefore $\operatorname{Ext}_{A}^{d}(M, A)$ is a graded simple $A^{\circ}$-module if and only if $M$ is a graded simple $A$-module. Thus we proved the AS-Gorenstein condition.

REMARK. A version of Theorem 1 holds for graded Hopf algebras. Here a graded Hopf algebra is slightly different from a graded algebra which is also a Hopf algebra. In a graded Hopf algebra the coalgebra structure is compatible with the $\mathbb{Z}_{2}$-grading and the Koszul sign convention applies for permuting symbols (see [5, pp. 205-6]). The proof of the statement is similar to the proof of Theorem 1.

ACKNOWLEDGEMENT. The first author was supported in part by the NSFC (project 10171016) and the second author was supported in part by the NSF.

\section{REFERENCES}

1. M. Artin and J. J. Zhang, Noncommutative projective schemes, Adv. Math. 109 (1994), $228-287$.

2. K. A. Brown, Representation theory of Noetherian Hopf algebras satisfying a polynomial identity, in Trends in the representation theory of finite-dimensional algebras (Seattle, WA, 1997), Contemp. Math. 229 (AMS, Providence, RI, 1998), 49-79.

3. K. A. Brown and K. R. Goodearl, Homological aspects of Noetherian PI Hopf algebras and irreducible modules of maximal dimension, J. Algebra 198 (1997), 240-265.

4. D. Chan, Noncommutative rational double points, MIT Thesis, 1999.

5. S. Montgomery, Hopf algebras and their actions on rings, CBMS Regional Conference Series in Mathematics 82 (Providence, RI, 1993).

6. M. Van den Bergh, Existence theorems for dualizing complexes over non-commutative graded and filtered rings, J. Algebra 195 (1997), 662-679.

7. Q.-S. Wu and J. J. Zhang, Homological identities for noncommutative rings, J. Algebra 242 (2001), 516-535.

8. Q.-S. Wu and J. J. Zhang, Dualizing complexes over noncommutative local rings, J. Algebra, 239 (2001), 513-548.

9. Q.-S. Wu and J. J. Zhang, Noetherian PI Hopf Algebras are Gorenstein, Trans. Amer. Math. Soc., to appear.

10. A. Yekutieli, Dualizing complexes over noncommutative graded algebras, J. Algebra 153 (1992), 41-84.

11. A. Yekutieli and J. J. Zhang, Rings with Auslander dualizing complexes, J. Algebra 213 (1999), 1-51. 